Table 4 Parameter Values and Standard Errors for Both Models over 100 Computed Growths up to 1,600 Abnormal Cells

\begin{tabular}{|c|c|c|c|c|c|c|c|}
\hline \multirow{3}{*}{ Value of $\kappa$} & \multicolumn{3}{|c|}{ D-M model (4) } & \multirow{3}{*}{$\begin{array}{l}\text { Standard } \\
\text { error } \\
s\end{array}$} & \multicolumn{3}{|c|}{ W-B model (3) } \\
\hline & & Parameter values & & & & eter values & $\begin{array}{l}\text { Standard } \\
\text { error }\end{array}$ \\
\hline & $A$ & $B$ & $C$ & & $\alpha$ & $\beta$ & $s$ \\
\hline$\infty$ (250 growths) & $0.23790(0.23914)$ & $0.51964(0.50693)$ & -0.41495 & 0.00255 & 0.45323 & $0.39039(0.3903)$ & 0.03088 \\
\hline 50 & $0.24052(0.24136)$ & $0.53494(0.52248)$ & -0.43151 & 0.00547 & 0.45303 & $0.39621(0.3956)$ & 0.03325 \\
\hline 20 & $0.24241(0.24486)$ & $0.56032(0.54703)$ & -0.44599 & 0.00885 & 0.45091 & $0.40801(0.4040)$ & 0.03591 \\
\hline 10 (98 growths) & $0.25097(0.25121)$ & $0.59442(0.59158)$ & -0.47121 & 0.00733 & 0.4497 & $0.42778(0.4192)$ & 0.03561 \\
\hline 5 (96 growths) & $0.26926(0.2663)$ & $0.65041(0.69740)$ & -0.51644 & 0.00526 & 0.44924 & $0.46198(0.4554)$ & 0.04116 \\
\hline 3 (82 growths) & $0.29815(0.29346)$ & $0.87830(0.88786)$ & -0.90934 & 0.00541 & 0.45967 & $0.48626(0.5204)$ & 0.11298 \\
\hline 2 (81 growths) & $0.34484(0.34777)$ & $1.28406(1.26880)$ & -1.21705 & 0.02291 & 0.44351 & $0.66475(0.6505)$ & 0.13434 \\
\hline
\end{tabular}

"Growths" which regress to zero after one step are not included. $A, B$ and $\beta$ were fitted to $\lambda+\mu /(\kappa-1)$ using least squares; the fitted values are given in parentheses.

If $E\left[T_{n} \mid \kappa\right]$ is the expected time to $n$ abnormal cells for a given $\kappa$, and if we conjecture that $E\left[1 / l_{n} \mid \kappa\right] \leq E\left[1 / l_{n} \mid \infty\right]$, then using the theory of recurrent events the ratio $E\left[T_{n} \mid \kappa\right] / E\left[T_{n} \mid \infty\right]$ can be shown to lie in the range $[1,1+2 /(\kappa-1)]$. Thus the replacement of $\beta$ in equation (4) by (constant $/(\kappa-1)$ ) is invalid.

The parameters $A, B$ and $\beta$ in equations (3) and (4) are obviously functions of $\kappa$, the forms of which we have been unable to establish theoretically. $A, B$ and $\beta$ have, however, been fitted, using least squares, to $\lambda+\mu / \kappa-1)$ and the fitted values are given in parentheses in Table 4.

In conclusion, it seems reasonable to suppose that the growth function tends to a function of the form $A \sqrt{ } n$ if large enough values of $n$ are considered. Our results are in agreement with Williams and Bjerknes and remain so for $n$ as large as 10,000 . If, however, the "transient" part of the growth function is extracted by considering variables such as $\left(T_{n}-\right.$ $\left.T_{1,600}\right)$ for each growth, we approach the "steady state" situation and $\alpha$ tends to 0.5 .

D. Y. Downham

R. K. B. MORGAN

Department of Computational and Statistical Science, Liverpool University

Received December 27, 1972.

1 Williams, T., and Bierknes, R., Nature, 236, 19 (1972).

2 Morgan, R. W., and Walsh, D. J. A., J. Roy. Statist. Soc., B, 27, 497 (1965).

${ }^{3}$ Hammersley, J. M., J. Roy. Statist. Soc., B, 28, 491 (1966).

\section{Renal Blood Flow in a Diving Trained Sea Lion}

THE diving reflex described for many species of animals involves a reduction in heart rate and the redistribution of blood flow to various tissues. Available evidence ${ }^{1,2}$ indicates that cardiac output decreases and that arterial pressure increases only modestly or remains unchanged during a dive. Flow measurements using restrained animals have tended to show cessation of flow to the kidney ${ }^{3,4}$ with diving. But it is well accepted that differences in these responses will exist with restrained and frightened animals as opposed to trained or free ranging animals. We investigated peripheral blood flow in a trained sea lion (Zalophus californiaus) by measuring the renal flow response to face immersion in water. Several months before surgery, the female sea lion was trained to place her head into a bucket, either empty or filled with water, and to remain in that position until otherwise commanded. During training, the animal wore a harness to which the necessary electronics were later attached.

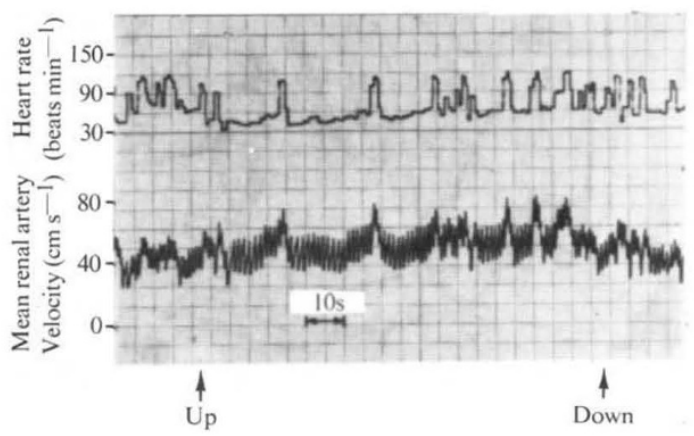

Fig. 1 Heart rate and mean renal artery flow velocity during breath holding or resting condition.

The animal was anaesthetized using a mixture of oxygen and fluothane after intramuscular injection of $0.020 \mathrm{mg} \mathrm{kg} \mathrm{kg}^{-1}$ atropine. An $8 \mathrm{mHz}$ Doppler flow cuff was implanted on the left renal artery under sterile surgical conditions. Care was exercised in the dissection of the artery so that minimal damage would be done to the perivascular renal nerves. The lead wires were tunnelled under the skin to the dorsal surface of the neck at which point they were brought through the skin. The mid-line incision was closed with interrupted sutures. For the next seven days, the animal was given oral antibiotics. Training was resumed about four days after surgery, but experiments were not performed until four weeks after the operation. The Doppler flow meter used followed the design of Franklin 5 . Recordings were made of mean renal artery flow velocity and heart rate using the flow velocity pulse to trigger the cardiotachometer. Jugular vein blood samples were obtained both pre- and post-operatively for red and white cell counts.

We show a representative portion of a control recording in Fig. 1. The resting heart rate on this occasion was 75 beats $\min ^{-1}$ and the control velocity was $45 \mathrm{~cm} \mathrm{~s}^{-1}$. The "down" arrow indicates the immersion of the animal's head into an empty bucket and the "up" arrow indicates the reverse. Breath holding periods can be observed with the heart rate as 
low as 36 beats $\min ^{-1}$ and the mean renal artery velocity varying with the rate. Thus breath holding in this situation was part of the effect of training. The average resting heart rate was 85 beats $\min ^{-1}$ and the average resting mean renal artery velocity was $48 \mathrm{~cm} \mathrm{~s}^{-1}$ for this animal during all of the experimental periods.

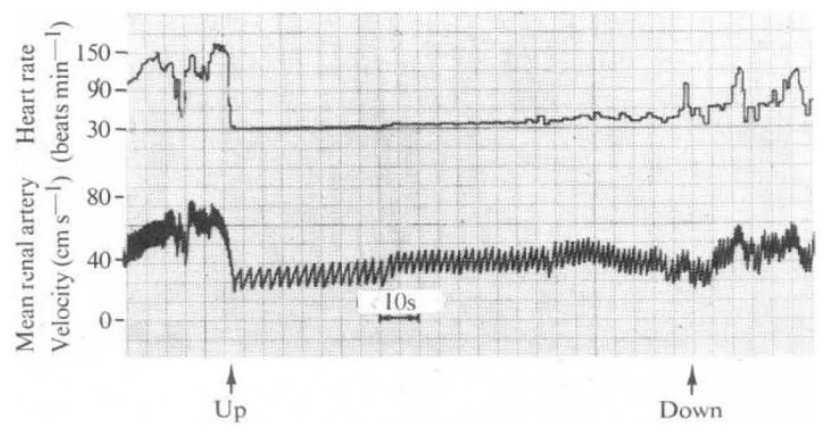

Fig. 2 Heart rate and mean renal flow velocity during face immersion for $2 \mathrm{~min}$.

The record shown in Fig. 2 was obtained during an immersion in water lasting $2 \mathrm{~min}$. A respiratory sinus arrhythmia occurred prior to immersion (right hand edge Fig. 2). Upon immersion a striking bradycardia began, with nadir of 30 beats $\min ^{-1}$. A reduction in mean renal artery velocity to $28 \mathrm{~cm} \mathrm{~s}^{-1}$ also occurred with some over-shoot in flow at the termination of the dive. The average length of 16 dives by the animal was $80 \mathrm{~s}$. The mean heart rate at the end of the dive was 34 beats $\min ^{-1}$ and the mean renal artery velocity was $31 \mathrm{~cm} \mathrm{~s}^{-1}$, which represents an average reduction of $34 \%$ below the control value. The health status of the animal as evaluated by red and white cell counts from jugular venous blood were almost identical. Because velocity is related to volume flow by the cross-sectional area of the vessel, which is fixed in the present experiment, inferences about flow can be made.

From the studies in this unrestrained animal, it seems that inflow to the kidney does not cease but is reduced together with the cardiac output as has been shown in the duck ${ }^{6}$. We offer three possible explanations for the variance from previous studies: First, intrarenal redistribution of blood flow; second, species difference; third, fright. Intrarenal redistribution of flow has been demonstrated in the kidney of non-diving mammals ${ }^{7,8}$. There is no way to rule out species difference; but fright is known to produce severe reaction in all animals. The record (Fig. 2) also demonstrates that there is an immediate reduction in flow, as occurs with cardiac output; thus these reductions are of neural origin. The delayed reduction in renal flow and the post-dive overshoot of the flow response are probably related to the duration of the dive. Afferent signals of renal origin may influence the duration of diving. Therefore, oxygen may be conserved in the kidney by shunting blood around highly metabolic areas such as cortex, and simultaneously these shunts may be used to adjust vascular resistance to ensure adequate perfusion of the heart and brain and to reduce the work of the heart specifically.

We thank Messrs Gerald Todd and Jerry Floyd for assistance.

\section{H. L. STONE \\ KEN GRAY \\ ROBERT STABE \\ J. M. ChANDLER, JUn.}

Marine Biomedical Institute,

University of Texas Medical Branch at Galveston,

200 University Boulevard,

Galveston, Texas 77550
Received November 13, 1972.

${ }^{1}$ Murdaugh, jun., H. V., Robin, E. D., Miller, J. E., Drewry, W. F., and Weiss, E., Amer. J. Physiol., 210, 176 (1966).

2 Elsner, R. W., Franklin, D. L., and Van Citters, R. L., Nature, 202, 809 (1964).

${ }^{3}$ Murdaugh, jun., H. V., Schmidt-Nielsen, B., Ward, J. W., and Mitchell, W. L., J. Cell Comp. Physiol., 58, 261 (1961).

${ }_{4}$ Bron, K. M., et al., Science, 152, 540 (1966).

5 Franklin, D. L., Schlegel, W. A., and Rushner, R. F., Science, 134, 564 (1961).

6 Johansen, K., Acta Physiol. Scand., 62, 1 (1964).

7 Pomeranz, B. H., Birtch, A. G., and Barger, A. C., Amer. J. Physiol., 215, 1067 (1968).

8 Hollenberg, N. K., Epstein, M., Guttman, R. D., Conroy, M., Basch, R. I., and Merrill, J. P., J. Appl. Physiol., 28, 312 (1970).

\section{Protection against Enteric Disease caused by Escherichia coli-a Model for Vaccination with a Virulence Determinant?}

AlthOUGH vaccination has assisted the control of many bacterial diseases, parenteral vaccination against enteric disease is not as satisfactory as we could wish ${ }^{\mathbf{1}}$. This is attributable to incomplete knowledge of both the pathogenesis of intestinal infections and the protective immune responses of the alimentary tract, with the result that vaccine development has been largely empirical. New knowledge of the specific determinants of microbial pathogenicity ${ }^{2}$ provides a sounder basis for the development of effective vaccines and the following report is an example of this approach to disease control.

The pathogenesis of porcine neonatal diarrhoea involves colonization of the intestine by an enteropathogenic strain of Escherichia coli $^{3}$ followed by production of enterotoxins ${ }^{4-7}$. Most strains of $E$. coli that cause neonatal diarrhoea synthesize one of two closely related protein surface antigens designated $\mathrm{K} 88 \mathrm{ab}$ or $\mathrm{K} 88 \mathrm{ac}^{8-10}$. The $\mathrm{K} 88$ antigen has adhesive properties that seem to be essential for the virulence of K88-positive enteropathogenic strains of $E$. coli $^{11}$ and enable K88-positive bacteria to proliferate in the anterior small intestine ${ }^{12}$. Thus, the two important virulence determinants of these strains of $E$. coli are (i) $\mathrm{K} 88$ antigen, which enables the bacteria to attach and attain large numbers in the small intestine, and (ii) enterotoxins, which cause severe diarrhoea, resulting in dehydration and death.

From these observations, we deduced that piglets could perhaps be protected against neonatal diarrhoea either by preventing bacterial attachment to the intestinal wall or by neutralizing the effects of enterotoxins. Antibodies directed against enterotoxins may be protective ${ }^{13,14}$, but protection has also been observed which seemed to be antibacterial rather than anti-enterotoxic ${ }^{15,16}$. Attachment of K88-positive bacteria to piglet intestinal tissue can be demonstrated in vitro and is inhibited by $\mathrm{K} 88$ antisera $^{11}$. Therefore, the antibacterial activity described in vivo could be due to an anti-K88 factor which prevents bacterial adhesion. There is no detectable placental transfer of antibodies in the pig ${ }^{17,18}$; so we postulated that K88 antibodies transmitted via the colostrum to the piglet's intestinal tract should reduce attachment of K88positive enteropathogenic bacteria to the mucosa and render the organism less virulent.

We investigated this hypothesis by immunizing pregnant gilts with partially-purified $\mathrm{K} 88$ antigens, then challenging their piglets with a virulent K88-positive strain of $E$. coli. $\mathrm{K} 88$ antigens were extracted from two laboratory-derived $\mathrm{K} 88^{+}$ strains [08:K27(A)-, K88ab:H- ${ }^{-}$and 08:K27(A) ${ }^{-}, \mathrm{K} 88 \mathrm{ac}$ : $\left.\mathrm{H}^{-}\right]$prepared by introducing the plasmids which determine 